\title{
Análise computacional da interação fluido-estrutura de uma tubulação de água utilizando o software comercial ANSYS
}

\author{
Computational analysis of the fluid-structure interaction of a water \\ pipe using ANSYS commercial software
}

Douglas S. Silva' ${ }^{1}$, Eduardo A. Nadai ${ }^{1}$, Viviane Ribeiro de Siqueira'

\section{RESUMO}

O objetivo deste trabalho é realizar um estudo de caso utilizando métodos computacionais e cálculos empíricos a fim de demonstrar que é possível realizar interações fluido-estrutura através de métodos numéricos e verificar sua eficiência realizando uma análise dos resultados. Os testes foram realizados utilizando as plataformas de softwares de modelagem 3D e simulação computacional, disponibilizadas pelo Laboratório de Sistemas Críticos do Parque Tecnológico São José dos Campos. O trabalho consiste na aplicação de diferentes pressões e velocidades na entrada e na saída de um modelo de tubulação de rede de distribuição de água residencial e observar as velocidades do fluido e as pressões que o escoamento exerce sob o modelo, a fim de verificar a perda de carga, a variação de pressão, a tensão e a deformação. Os resultados computacionais obtidos neste artigo estão em concordância com os valores empíricos, confirmando assim que é possível realizar simulações numéricocomputacionais da interação fluido-estrutura.

Palavras-chave: Simulação numérica; Interação fluido-estrutura; Mecânica dos fluidos computacional.

\begin{abstract}
The purpose of this work is a case study using computational methods and empirical calculations to demonstrate that it is possible to perform fluid-structure interaction by numerical method and check its efficiency by performing an analysis of the results. The tests were performed using the 3D modeling software platforms and computer simulation provided by the Laboratório de Sistemas Críticos do Parque Tecnológico São José dos Campos. The work involves the application of different pressures and velocities at the inlet and outlet of a pipe network model of residential water distribution and observe the speed of the fluid and the pressure that the flow exerts on the model in order to verify the loss load, the pressure variation, stress and deformation. The computational results obtained in this article are in agreement with the empirical values, thus confirming that it is possible to perform numerical-computational simulations of the fluid-structure interaction.
\end{abstract}

Keywords: Numerical simulation; Fluid structure interaction; Computational fluid mechanic.

${ }^{1}$ Faculdade de Tecnologia de São José dos Campos Prof. Jessen Vidal - São José dos Campos (SP) - Brasil

Autor correspondente: Douglas S. Silva - Faculdade de Tecnologia de São José dos Campos Prof. Jessen Vidal - Av. Cesare Mansueto Giulio Lattes, s/n - Distrito de Eugenio de Melo - CEP: 12247-014 - São José dos Campos (SP) - Brasil

E-mail: douglas.silva52@fatec.sp.gov.br

Recebido: 23/01/2017 Aprovado: 22/03/2017 


\section{INTRODUÇÃO}

O dimensionamento de sistemas hidráulicos - posicionamento, escolha do material e acessórios - são aspectos de suma importância para o desenvolvimento de um projeto hidráulico, seja ele o sistema de combustível de um avião, o sistema hidráulico de uma casa ou até mesmo de uma grande tubulação de gás encanado. Para determinar esses aspectos, especialistas utilizam métodos empíricos, que são os cálculos convencionais disponíveis na literatura. As equações dependem de diversos parâmetros como pressão, vazão, densidade, que variam dependendo das características específicas do fluido e do material que será utilizado para a construção dos tubos ${ }^{(1)}$.

De acordo com Wylie ${ }^{(2)}$, com o avanço dos computadores e a utilização de poderosas ferramentas computacionais, é possível realizar simulações numéricas que nos permitem analisar diversos tipos de modelos e situações de maneira rápida e eficaz, permitindo ao especialista eleger a melhor situação para desenvolver seu projeto, antes mesmo de realizar qualquer ensaio real em laboratório, acarretando menores custos de fabricação e melhor desempenho do projeto. De acordo com Fortuna ${ }^{(3)}$, estas ferramentas computacionais estão sendo cada vez mais empregadas na indústria por atender a uma vasta gama de setores, entre eles aeronáutico, engenharia ambiental, engenharia costeira, medicina, siderurgia, indústria têxtil, entre outros, e permite ao especialista resolver problemas através de simulações estruturais e de fluidodinâmica e interações entre diferentes estados físicos da matéria conhecida como análise fluido-estrutura.

Neste trabalho demonstramos como podemos utilizar simulações fluido-estrutura para realizar análises em modelos de tubulação de redes de distribuição de água residencial para diferentes valores de velocidade e pressão de escoamento a fim de obter como resultado a perda de carga e os perfis de pressão, tensão e deformação exercida nas paredes do duto.

\section{METODOLOGIA}

Antes de iniciar as simulações numéricas, foram realizados cálculos do número de Reynolds para identificar o tipo de escoamento. Depois, realizaram-se cálculos para determinar a velocidade do fluido no interior do tubo e a tensão na parede do tubo, exercida pela pressão do fluido. Também foram calculadas as perdas de carga.

Para gerar a geometria do modelo foi utilizado o software comercial CATIA V5 e para o processo de simulação numérica os softwares ANSYS CFX 17.2, para as análises fluidodinâmicas (método dos volumes finitos), e ANSYS MECHANICAL 17.2, para as análises mecânicas (método dos elementos finitos).

O modelo obedeceu a dimensões reais de uma tubulação de água fria ${ }^{(4)}$, representada de maneira simplificada na Fig. 1. O modelo é composto por 2 tubos lineares de $0,5 \mathrm{~m}$ de comprimento cada e diâmetro interno de $0,02 \mathrm{~m}$, unidos por uma curva de $90^{\circ}$ e raio de $0,04 \mathrm{~m}$.

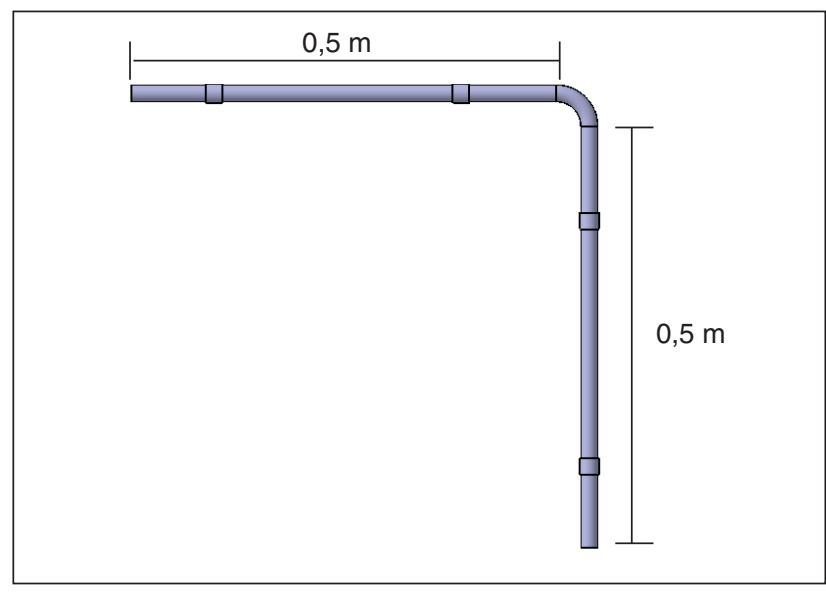

Figura 1: Modelo CAD 2D - Tubo de 0,02 m de diâmetro.

\section{Método Analítico}

A validação de resultados pode ser realizada com o emprego de técnicas numéricas, análises teóricas e métodos experimentais ${ }^{(3)}$. No atual trabalho, foram realizadas simulações numéricas e soluções analíticas. De acordo com Post ${ }^{(5)}$, o escoamento interno em tubos pode ser considerado laminar quando o número de Reynolds obtido é igual ou menor que 2000. Através da Eq. 1 é possível identificar o tipo de escoamento presente na tubulação em estudo:

$$
R e=\frac{\rho V L}{\mu}
$$

onde: $\mathrm{Re}=$ Número de Reynolds; $\mathrm{V}=$ Velocidade média do fluido $(\mathrm{m} / \mathrm{s}) ; \mathrm{L}=$ Diâmetro interno do tubo $(\mathrm{m}) ; \rho=$ Massa específica do fluido $\left(\mathrm{kg} / \mathrm{m}^{3}\right)$ e $\mu=$ Viscosidade dinâmica do fluido (N.s $\left./ \mathrm{m}^{2}\right)$.

Segundo Post ${ }^{(5)}$, pode-se determinar a velocidade no interior de um duto através da Eq. 2:

$$
V=\sqrt{2 \frac{\Delta P}{\rho}}
$$

onde: $\mathrm{V}=$ Velocidade $(\mathrm{m} / \mathrm{s}) ; \Delta \mathrm{P}=$ Diferencial de pressão $(\mathrm{Pa})$; $\rho=$ Massa específica do fluido $\left(\mathrm{kg} / \mathrm{m}^{3}\right)$.

A tensão na parede do tubo pode ser encontrada utilizando a Eq. $3^{(6)}$ :

$$
\sigma=\frac{P r}{e}
$$

onde: $\sigma=$ Tensão na parede do tubo $(\mathrm{Pa}) ; \mathrm{P}=$ Pressão $(\mathrm{Pa})$; $\mathrm{r}=$ Raio interno do tubo $(\mathrm{m}) ; \mathrm{e}=$ Espessura da parede do tubo $(\mathrm{m})$.

De acordo com Post ${ }^{(5)}$, para se determinar perda de carga por atrito para escoamento em regime estacionário utiliza-se a Eq. 4:

$$
J=f \frac{L}{D} \frac{V^{2}}{2 g}
$$

onde: $\mathrm{J}=$ Perda de carga $(\mathrm{m}) ; \mathrm{f}=$ Fator de atrito; $\mathrm{L}=$ Comprimento total do tubo (m); $\mathrm{D}=$ Diâmetro interno do tubo (m); 
$\mathrm{V}=$ Velocidade do fluido $(\mathrm{m} / \mathrm{s}) ; \mathrm{g}$ = Aceleração da gravidade $\left(9,81 \mathrm{~m} / \mathrm{s}^{2}\right)$.

Para escoamentos laminares, o fator de atrito pode ser obtido através da razão 64/Re. Para escoamento turbulento, o fator de atrito foi obtido utilizando o Diagrama de $\operatorname{Moody}^{(7)}$.

\section{Análise Fluidodinâmica}

Nas simulações fluidodinâmicas a água foi utilizada como o fluido de trabalho, em temperatura igual a $20^{\circ} \mathrm{C}$. Para análise comparativa entre método analítico e numérico, a fim de validar as simulações realizadas, efetuou-se um estudo de perda de carga a uma velocidade inicial de $0,1 \mathrm{~m} / \mathrm{s}$. O resultado da validação é apresentado na Tabela 1. Após a análise comparativa, foram realizadas doze diferentes rodadas de simulações onde a pressão absoluta inicial aplicada variou a cada simulação, com valores entre $5 \mathrm{kPa}$ e $850 \mathrm{kPa}$. Foi utilizado esse range de pressão a fim de simular o comportamento do fluido em condições reais de escoamento e, com a finalidade de realizar um estudo numérico, utilizaram-se altos valores de pressão para observar o comportamento do material da tubulação. Importante ressaltar que a partir de $200 \mathrm{kPa}$ a tubulação real pode se romper. Variouse também a velocidade na saída de 0,1 a $3 \mathrm{~m} / \mathrm{s}$ para obter a variação de pressão no duto. Também foram considerados valores para aceleração da gravidade de $9,81 \mathrm{~m} / \mathrm{s}^{2}$, e pressão atmosférica ao nível do mar igual a $101,3 \mathrm{kPa}$. As paredes do tubo foram definidas com condição de "no slip wall". Como critério de convergência, definimos o valor de resíduo de cálculo RMS (Root Mean Square) igual ou inferior a $10^{-4}$, atingido em todas as simulações. Na Fig. 2 pode-se visualizar a direção do escoamento aplicado.

Tabela 1: Perda de carga.

\begin{tabular}{|c|c|c|}
\hline$V_{e}(m / s)$ & $J_{a}(m)$ & $J_{n}(m)$ \\
\hline 0,1 & 0,00279 & 0,00247 \\
\hline
\end{tabular}

$\mathrm{V}_{\mathrm{e}}=$ Velocidade na entrada do duto; $\mathrm{J}_{\mathrm{a}}=$ Perda de carga analítica e $\mathrm{J}_{\mathrm{n}}=$ Perda de carga numérica $(m)$.

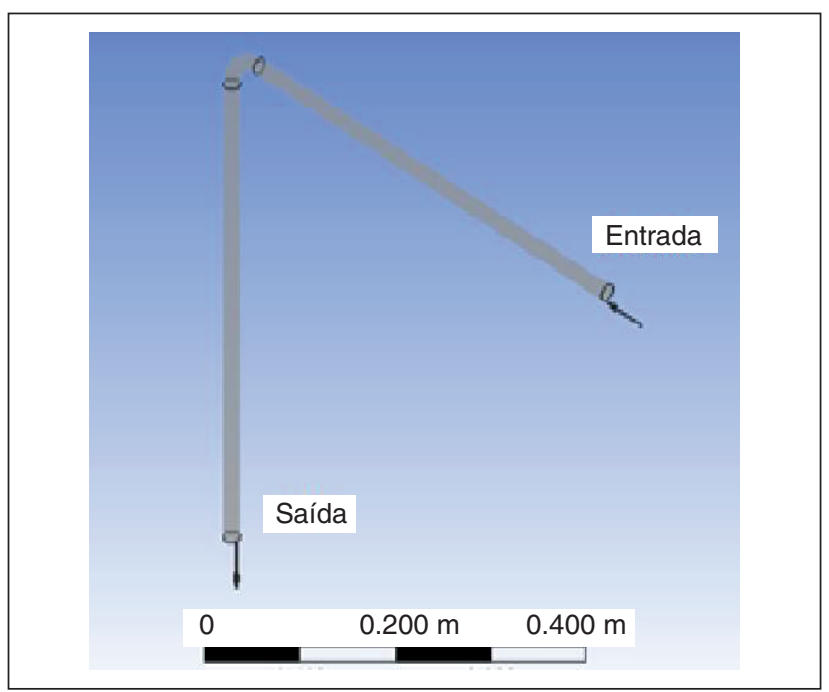

Figura 2: Direção de escoamento.

\section{Análise Mecânica}

Para a análise mecânica, o material do tubo utilizado para as simulações é o PVC (Poly Vinyl Chloride) de características mecânicas descritas em catálogo comercial de produto $^{(8)}$.

A interação fluido-estrutura é realizada através da ferramenta “importedload”, já incorporada no software ANSYS. Através desta ferramenta é possível importar a pressão atuante nas paredes da tubulação, pressão esta que é resultante do escoamento anteriormente simulado. Na Fig. 3 observa-se os valores mínimo e máximo de pressão na parede do tubo 5,1986E-6 MPa e 0,10457 $\mathrm{MPa}$, respectivamente. Para essa análise, foi definida como condição de contorno a pressão de entrada igual a $200 \mathrm{kPa}$.

A fixação do tubo para a análise estrutural estática foi realizada pelas abraçadeiras destacadas em verde na Fig. 4.

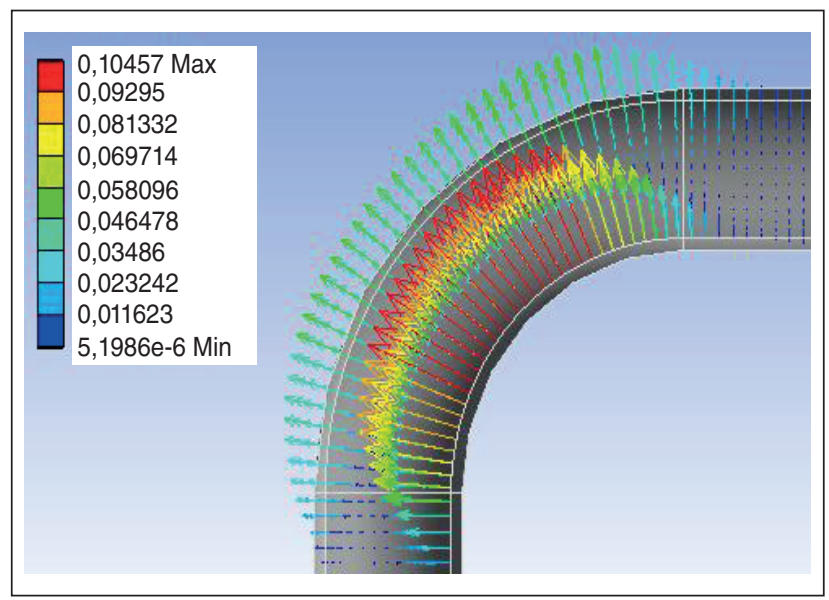

Figura 3: Pressão resultante do escoamento a $200 \mathrm{kPa}$.

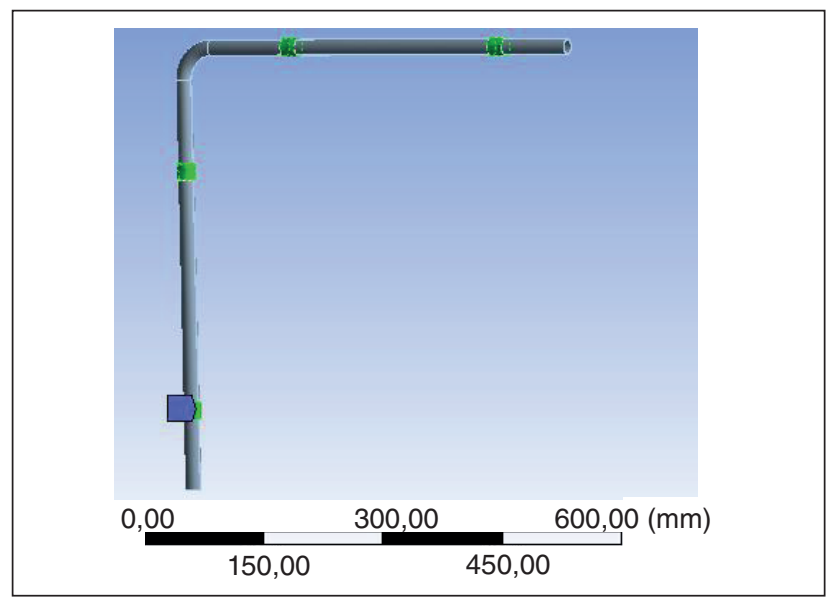

Figura 4: Fixação tubo, análise estrutural.

\section{RESULTADOS}

Aplicando uma velocidade de $0,1 \mathrm{~m} / \mathrm{s}$ como condição de contorno na entrada do tubo, compararam-se os resultados de perda de carga obtidos através dos métodos numérico e analítico.

Devido à perda de carga, há uma variação da velocidade na saída e, com a variação da velocidade, tem-se uma variação 
da pressão. A variação de pressão é dada pela diferença entre a pressão de entrada e a pressão da saída, demonstrada na Fig. 5.

Na Fig. 6 observa-se o perfil de velocidades do fluido no trajeto do duto com velocidade de saída de $1 \mathrm{~m} / \mathrm{s}$.

Para realizar a análise fluido-estrutura, doze diferentes pressões na entrada do tubo foram aplicadas a fim de obter os perfis de pressão e as tensões resultantes na parede do duto. A Fig. 7 mostra

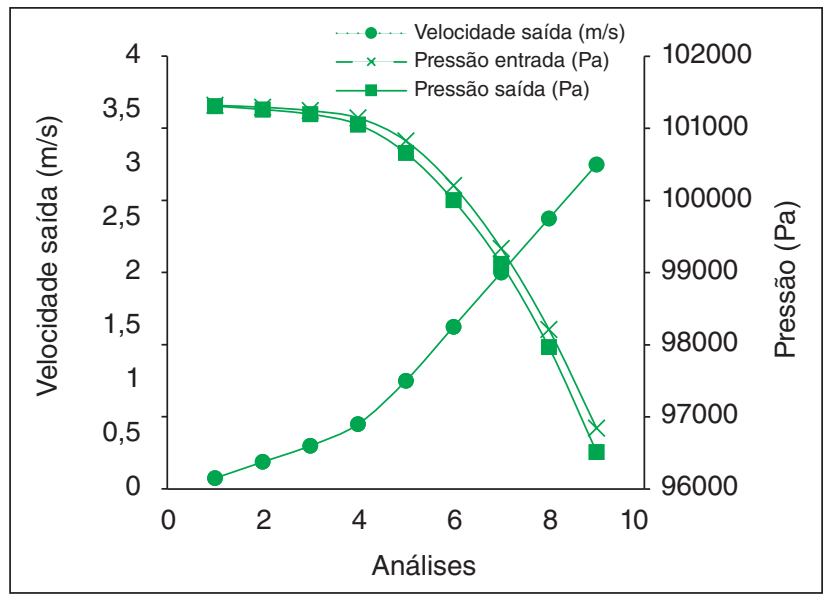

Figura 5: Variação da pressão com relação a velocidade de saída.

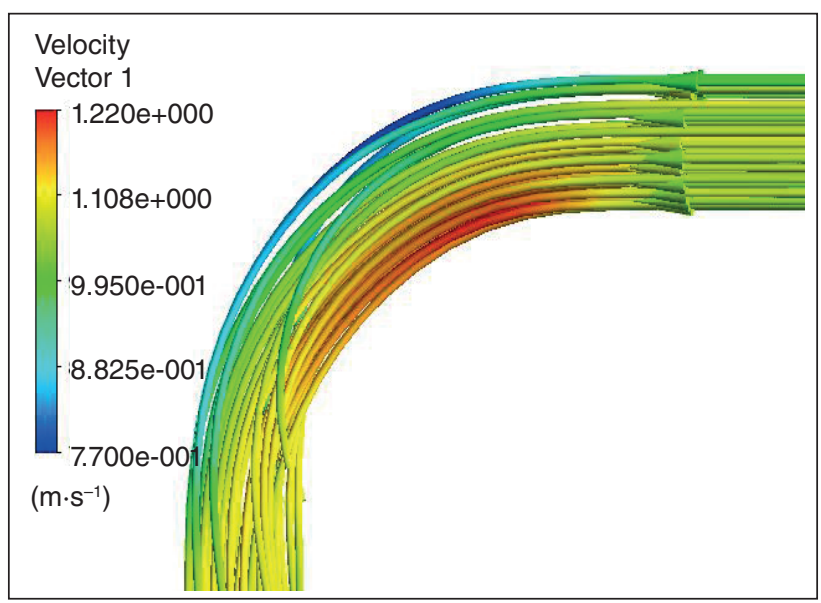

Figura 6: Perfil de escoamento de velocidade $(\mathrm{m} / \mathrm{s})$.

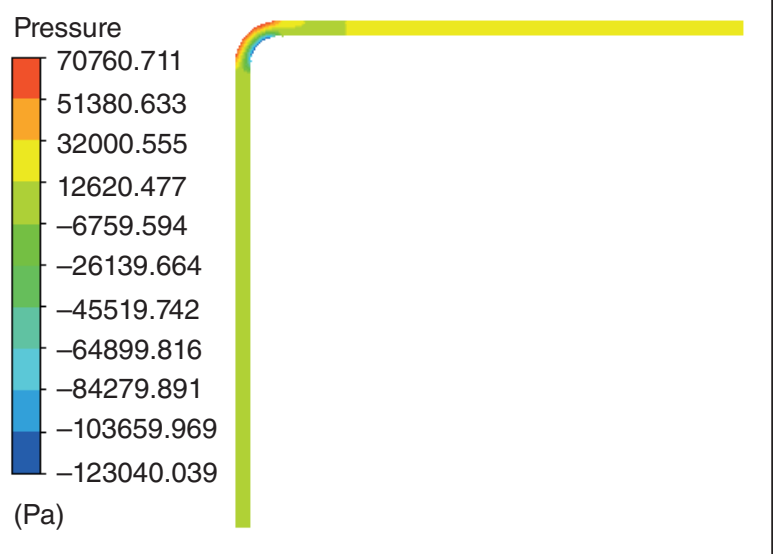

Figura 7: Perfil de pressão (Pa). o perfil de pressão do fluido considerando a pressão na entrada igual a $200 \mathrm{kPa}$.

A Tabela 2 tem como objetivo demonstrar alguns dos resultados das tensões exercidas nas paredes do tubo calculadas pelo método analítico e numérico decorrentes à pressão aplicada pelo fluido.

Tabela 2: Tensões resultantes na parede do tubo.

\begin{tabular}{c|c|c|}
\hline $\mathrm{Pi}(\mathrm{kPa})$ & $\sigma_{\mathrm{a}}(\mathrm{MPa})$ & $\sigma_{\mathrm{n}}(\mathrm{MPa})$ \\
\hline 150 & 1 & 0,9974 \\
200 & 1,3333 & 1,3323 \\
650 & 4,3333 & 4,3664 \\
750 & 5 & 5,0409 \\
850 & 5,6667 & 5,7156 \\
\hline
\end{tabular}

$P_{i}=$ Pressão na entrada do duto; $\sigma_{a}=$ Tensão analítica e $\sigma_{n}=$ Tensão numérica.

Segundo $\mathrm{Netto}^{(7)}$, em condições reais com pressões a partir de $200 \mathrm{kPa}$ pode ocorrer a ruptura do material. Submetido a pressões inferiores o material não se rompe, mas pode sofrer alguma deformação. A Fig. 8 mostra a deformação elástica (deformação não permanente) nas paredes do tubo decorrente à tensão exercida pelo escoamento com pressão na entrada de $200 \mathrm{kPa}$.

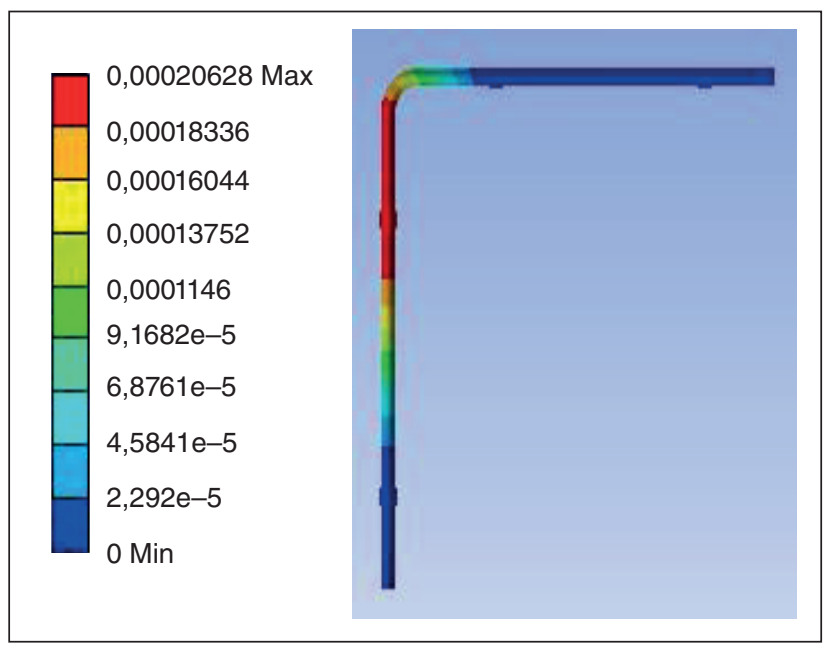

Figura 8: Deformação elástica nas paredes do tubo $(\mathrm{m})$.

\section{CONCLUSÃO}

Ao término deste estudo, conclui-se que é possível realizar análises fluido-estrutura por método computacional, análises essas que só foram possíveis graças a evolução dos softwares de simulação e capacidade computacional. Concluiu-se também que análises numéricas possuem uma boa eficiência para análises como perda de carga, variação de pressão e tensão.

Para modelagem da geometria, geração da malha, definições do modelo físico - condições iniciais e condições de contorno - e pós-processamento, foram necessárias 10 horas de trabalho.

Para a realização das simulações, utilizou-se um computador com um processador de $3.30 \mathrm{GHz}$. Devido à capacidade de processamento disponibilizada, foram necessários 72 minutos de 
processamento para a realização de todas as simulações.Para fim comparativo, executamos as mesmas simulações em um cluster que conta com 60 núcleos de processamento com $2.40 \mathrm{GHz}$ e o tempo necessário para conclusão foi de 8 minutos.

\section{AGRADECIMENTOS}

Agradecemos ao Parque Tecnológico São José dos Campos por disponibilizar a infraestrutura, os equipamentos e softwares do Laboratório de Sistemas Críticos. Agradecemos as professoras Viviane e Lise da FATEC São José dos Campos por apoiar o desenvolvimento deste trabalho. Através dos mesmos este trabalho pode ser realizado com grande qualidade, contribuindo para o crescimento intelectual dos autores deste.

\section{REFERÊNCIAS}

1. SeVZATIAN, L.; PIMENTA, F. Manual de Escopo de Projetos e Serviços de Hidráulica. São Paulo, ABRASIP, 2012.

2. WYLIE, B. J. N. Aplication of Two-Dimensional Cellular Automaton Lattice-Gas Models to the Simulation of Hydrodynamics. Edinburgh: UniversityofEdingurgh, 1990.

3. FORTUNA, A. O. Técnicas Computacionais para Dinâmica dos Fluidos. São Paulo: Edusp, 2000.

4. TIGRE. LinhaSoldável. Joinville, 2013. (CatálogoComercial).

5. POST, S. Mecânica dos Fluidos Aplicada e Computacional. Rio de Janeiro: LTC, 2013

6. NASH, W. A. Resistência dos Materiais. São Paulo: Editora MacGraw-Hill, 1977.

7. NETTO, A. Z. et. al. Manual de Hidráulica. São Paulo: Blucher, 1998.

8. BRASKEM. Boletim Técnico. São Paulo, 2002. (Catálogo comercial). 